\title{
Evidence of BRST-Symmetry Breaking in Lattice Minimal Landau Gauge
}

\author{
Attilio Cucchieri* \\ Instituto de Física de São Carlos, Universidade de São Paulo, Caixa Postal 369, 13560-970 São \\ Carlos, SP, Brazil \\ E-mail: attiliodifsc.usp.br

\section{David Dudal} \\ KU Leuven Campus Kortrijk - KULAK, Department of Physics, Etienne Sabbelaan 53, 8500 \\ Kortrijk, Belgium \\ Ghent University, Department of Physics and Astronomy, Krijgslaan 281-S9, 9000 Gent, \\ Belgium \\ E-mail: david.dudal@ugent.be

\section{Tereza Mendes} \\ Instituto de Física de São Carlos, Universidade de São Paulo, Caixa Postal 369, 13560-970 São \\ Carlos, SP, Brazil \\ E-mail: mendes@ifsc.usp.br
}

\section{Nele Vandersickel}

Ghent University, Department of Physics and Astronomy, Krijgslaan 281-S9, 9000 Gent,

Belgium

E-mail: nele.vandersickel@ugent.be

\begin{abstract}
By evaluating the so-called Bose-ghost propagator, we present the first numerical evidence of BRST-symmetry breaking for Yang-Mills theory in minimal Landau gauge, i.e. due to the restriction of the functional integration to the first Gribov region in the Gribov-Zwanziger approach. Our data [1] are well described by a simple fitting function, which can be related to a massive gluon propagator in combination with an infrared-free (Faddeev-Popov) ghost propagator. As a consequence, the Bose-ghost propagator, which has been proposed as a carrier of the confining force in minimal Landau gauge, displays a $1 / p^{4}$ singularity in the infrared limit.
\end{abstract}

The 32nd International Symposium on Lattice Field Theory,

23-28 June, 2014

Columbia University New York, NY

${ }^{*}$ Speaker. 


\section{Introduction}

The search for confinement signatures in the infrared (IR) behavior of Green's functions is a longstanding holy grail of Yang-Mills theories and Quantum Chromodynamics. Indeed, as stressed by West [2], already the area-law criterion of Wilson [3] was partially based on the presumed IR behavior of the gluon propagator. This viewpoint was also addressed by several studies, based on functional approaches, such as the works of Mandelstam [4], Baker, Ball and Zachariasen [5, 6], Cornwall [7], Stingl [8], to name a few.

Among the possible gauge-fixing conditions considered for the evaluation of Green's functions in Yang-Mills theories, the so-called minimal Landau gauge has attracted a great deal of attention since Gribov's work [9]. Let us recall that, in this case, the gauge condition is implemented by restricting the functional integral over gauge-field configurations to the so-called first Gribov region $\Omega$, i.e. to the set of transverse configurations for which the Faddeev-Popov matrix $\mathcal{M}^{a b}(x, y)$ is non-negative. In a numerical simulation (on the lattice) this can be easily obtained by minimizing a suitable functional (see for example Ref. [10]). On the other hand, in analytic studies, this restriction is achieved by adding a nonlocal term $S_{\mathrm{h}}$, the horizon function, to the usual Landau gauge-fixed Yang-Mills action $S_{\mathrm{YM}}+S_{\mathrm{gf}}$. One thus obtains the Gribov-Zwanziger (GZ) action $S_{\mathrm{GZ}}=S_{\mathrm{YM}}+S_{\mathrm{gf}}+\gamma^{4} S_{\mathrm{h}}$, where the massive parameter $\gamma$, known as the Gribov parameter, is dynamically determined by a self-consistent condition, the horizon condition.

In order to localize the GZ action [11] one introduces a pair of complex-conjugate bosonic fields $\left(\bar{\phi}_{\mu}^{a c}, \phi_{\mu}^{a c}\right)$ and a pair of Grassmann complex-conjugate fields $\left(\bar{\omega}_{\mu}^{a c}, \omega_{\mu}^{a c}\right)$. Then, the GZ action can be written as $S_{\mathrm{GZ}}=S_{\mathrm{YM}}+S_{\mathrm{gf}}+S_{\mathrm{aux}}+S_{\gamma}$, where

$$
\begin{aligned}
S_{\text {aux }} & =\int \mathrm{d}^{4} x\left[\bar{\phi}_{\mu}^{a c} \partial_{v}\left(D_{v}^{a b} \phi_{\mu}^{b c}\right)-\bar{\omega}_{\mu}^{a c} \partial_{v}\left(D_{v}^{a b} \omega_{\mu}^{b c}\right)-g_{0}\left(\partial_{v} \bar{\omega}_{\mu}^{a c}\right) f^{a b d} D_{v}^{b e} \eta^{e} \phi_{\mu}^{d c}\right] \\
S_{\gamma} & =\int \mathrm{d}^{4} x\left[\gamma^{2} D_{v}^{b a}\left(\phi_{v}^{a b}+\bar{\phi}_{v}^{a b}\right)-4\left(N_{c}^{2}-1\right) \gamma^{4}\right] .
\end{aligned}
$$

Under the nilpotent BRST variation $s$, the four auxiliary fields form two BRST doublets, i.e.

$$
s \phi_{\mu}^{a c}=\omega_{\mu}^{a c}, \quad s \omega_{\mu}^{a c}=0
$$

and

$$
s \bar{\omega}_{\mu}^{a c}=\bar{\phi}_{\mu}^{a c}, \quad s \bar{\phi}_{\mu}^{a c}=0,
$$

giving rise to a BRST quartet. At the same time, one can check that the localized GZ theory is not BRST-invariant. Indeed, while $s\left(S_{\mathrm{YM}}+S_{\mathrm{gf}}+S_{\text {aux }}\right)=0$, one finds that $s S_{\gamma} \propto \gamma^{2} \neq 0$. Since a nonzero value for the Gribov parameter $\gamma$ is implied by the restriction of the functional integration to the Gribov region $\Omega$, it is clear that BRST-symmetry breaking is expected, as a direct consequence of this restriction.

\section{The Bose-Ghost Propagator}

In order to study numerically the effect of the BRST-breaking term $S_{\gamma}$, one can consider the expectation value of a BRST-exact quantity. For example, the correlation function

$$
Q_{\mu \nu}^{a b c d}(x, y)=\left\langle s\left(\phi_{\mu}^{a b}(x) \bar{\omega}_{v}^{c d}(y)\right)\right\rangle=\left\langle\omega_{\mu}^{a b}(x) \bar{\omega}_{v}^{c d}(y)+\phi_{\mu}^{a b}(x) \bar{\phi}_{v}^{c d}(y)\right\rangle
$$


should have a zero expectation value for a BRST-invariant theory, but it does not necessarily vanish if BRST symmetry is broken [12]. Indeed, one can verify [11, 13] that at tree level (and in momentum space)

$$
Q_{\mu \nu}^{a b c d}\left(p, p^{\prime}\right)=\frac{(2 \pi)^{4} \delta^{(4)}\left(p+p^{\prime}\right) g_{0}^{2} \gamma^{4} f^{a b e} f^{c d e} P_{\mu v}(p)}{p^{2}\left(p^{4}+2 g_{0}^{2} N_{c} \gamma^{4}\right)},
$$

where $P_{\mu v}(p)$ is the usual transverse projector. Thus, this propagator is proportional to the Gribov parameter $\gamma$, i.e. its nonzero value is clearly related to the breaking of the BRST symmetry in the GZ theory. One should also recall that this Bose-ghost propagator has been proposed as a carrier of long-range confining force in minimal Landau gauge $[14,15,16]$.

On the lattice one does not have direct access to the auxiliary fields $\left(\bar{\phi}_{\mu}^{a c}, \phi_{\mu}^{a c}\right)$ and $\left(\bar{\omega}_{\mu}^{a c}, \omega_{\mu}^{a c}\right)$. On the other hand, by 1) adding suitable sources to the GZ action, 2) explicitly integrating over the four auxiliary fields and 3) taking the usual functional derivatives with respect to the sources, ${ }^{1}$ one can verify that [14]

$$
Q_{\mu \nu}^{a b c d}(x-y)=\gamma^{4}\left\langle R_{\mu}^{a b}(x) R_{v}^{c d}(y)\right\rangle,
$$

where

$$
R_{\mu}^{a c}(x)=\int \mathrm{d}^{4} z\left(\mathcal{M}^{-1}\right)^{a e}(x, z) B_{\mu}^{e c}(z)
$$

and

$$
B_{v}^{b c}(x)=g_{0} f^{b e c} A_{v}^{e}(x) .
$$

\section{Numerical Simulations}

We evaluated [1] the Bose-ghost propagator, defined in Eq. (2.3) above, in momentum space -modulo the global factor $\gamma^{4}$ - using numerical simulations in the SU(2) case. In order to check discretization and finite-volume effects, we considered three different values of the lattice coupling $\beta$ and five different physical volumes, ranging from about $(3.366 \mathrm{fm})^{4}$ to $(10.097 \mathrm{fm})^{4}$. Numerical results for the scalar function $Q\left(k^{2}\right)$, defined through the relation [see Eq. (2.2)]

$$
Q^{a c}(k) \equiv Q_{\mu \mu}^{a b c b}(k) \equiv \delta^{a c} N_{c} P_{\mu \mu}(k) Q\left(k^{2}\right),
$$

are shown in Figs. 1 and 2 as a function of $p^{2}(k)$. [We indicate with $p(k)$ the lattice momentum with components $p_{\mu}=2 \sin \left(\pi k_{\mu} / N\right)$, where $N$ is the lattice side and $k$ is the wave vector with components $k_{\mu}=0,1, \ldots, N-1$.] Note that, in the latter case, we plot the scalar function $Q\left(k^{2}\right)$ multiplied by $p^{4}$, in order to make evident the IR behavior of the Bose-ghost propagator. The data scale quite well, even though small deviations are observable in the IR limit (see Fig. 2).

We also fit the data using the fitting function

$$
f\left(p^{2}\right)=\frac{c}{p^{4}} \frac{p^{2}+s}{p^{4}+u^{2} p^{2}+t^{2}} .
$$

\footnotetext{
${ }^{1}$ This is analogous to the evaluation of the Faddeev-Popov correlation function, i.e. the ghost propagator, for which, on the lattice, one uses the relation

$$
\left.\left\langle\bar{c}^{a}(x) c^{b}(y)\right)\right\rangle=\left\langle\left(\mathcal{M}^{-1}\right)^{a b}(x, y)\right\rangle .
$$
}




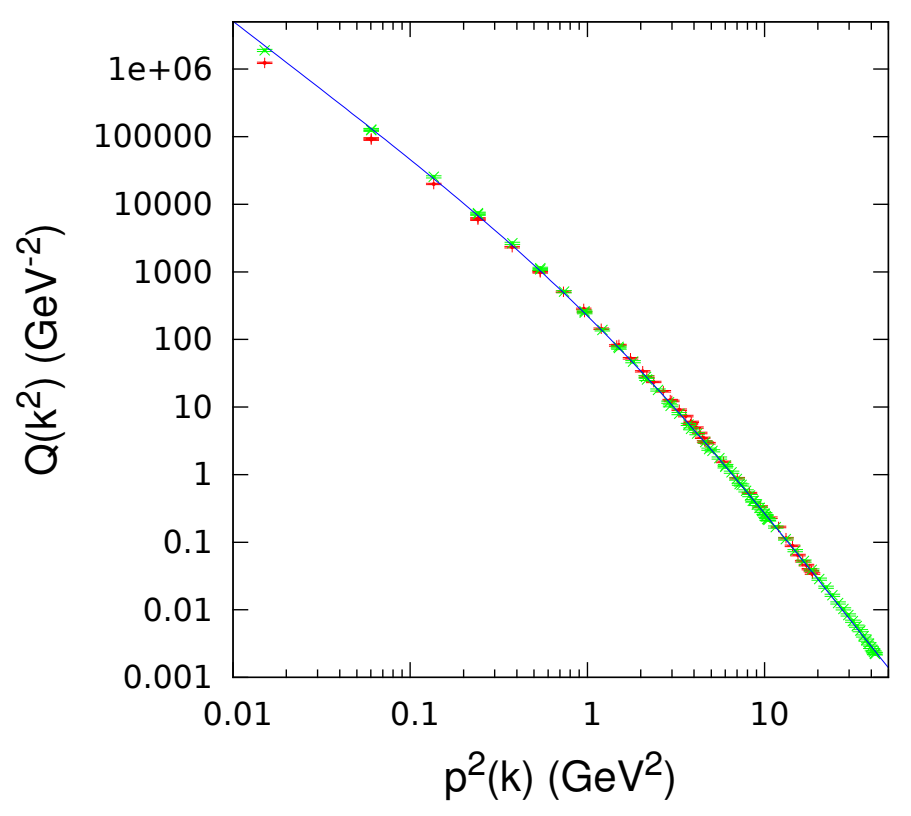

Figure 1: Data for $\beta=2.2$ and $V=48^{4}(+)$ matched $[17,18]$ with data for $\beta=2.34940204$ and $V=72^{4}$ $(\times)$, fitted using Eq. (3.2) with $t=3.2(0.3)\left(\mathrm{GeV}^{2}\right), u=3.6(0.4)(\mathrm{GeV}), s=46(13)\left(\mathrm{GeV}^{2}\right)$ and $c=114(13)$.

Following the analysis in $[14,16]$, this fitting function corresponds to considering an infrared-free (Faddeev-Popov) ghost propagator $G\left(p^{2}\right)$ and a massive gluon propagator $D\left(p^{2}\right)$. The fit describes the data quite well. As a consequence, the Bose-ghost propagator presents a $p^{-4}$ singularity in the IR limit. This result is in agreement with the one-loop analysis carried out in [19]. One should stress that, even though a double-pole singularity is suggestive of a long-range interaction, the above result does not imply a linearly-rising potential between quarks $[14,16,19]$. Indeed, when coupled to quarks via the $A-\phi$ propagator - which is nonzero due to the vertex term $\bar{\phi}_{\mu}^{a c} g f^{a c b} A_{v}^{c} \partial_{\nu} \phi_{\mu}^{b c}$ in Eq. (1.1) —, the Bose-ghost propagator gets a momentum factor at each vertex $[14,16]$, i.e. the effective propagator is given by $p^{-2}$ in the IR limit.

\section{Conclusions}

We presented the first numerical evaluation of the Bose-ghost propagator in minimal Landau gauge. We find that our data are well described by a simple fitting function, which can be related to a massive gluon propagator in combination with an IR-free (Faddeev-Popov) ghost propagator, implying a $p^{-4}$ singularity in the IR limit. Our results constitute the first numerical manifestation of BRST-symmetry breaking due to the restriction of the functional integration to the Gribov region $\Omega$ in the GZ approach. This directly affects continuum functional studies in Landau gauge, which usually employ lattice results as an input and/or as a comparison. At the same time, several questions are still open for a clear understanding of the GZ approach. In particular, one should understand how a physical positive-definite Hilbert space could be defined in this case. 


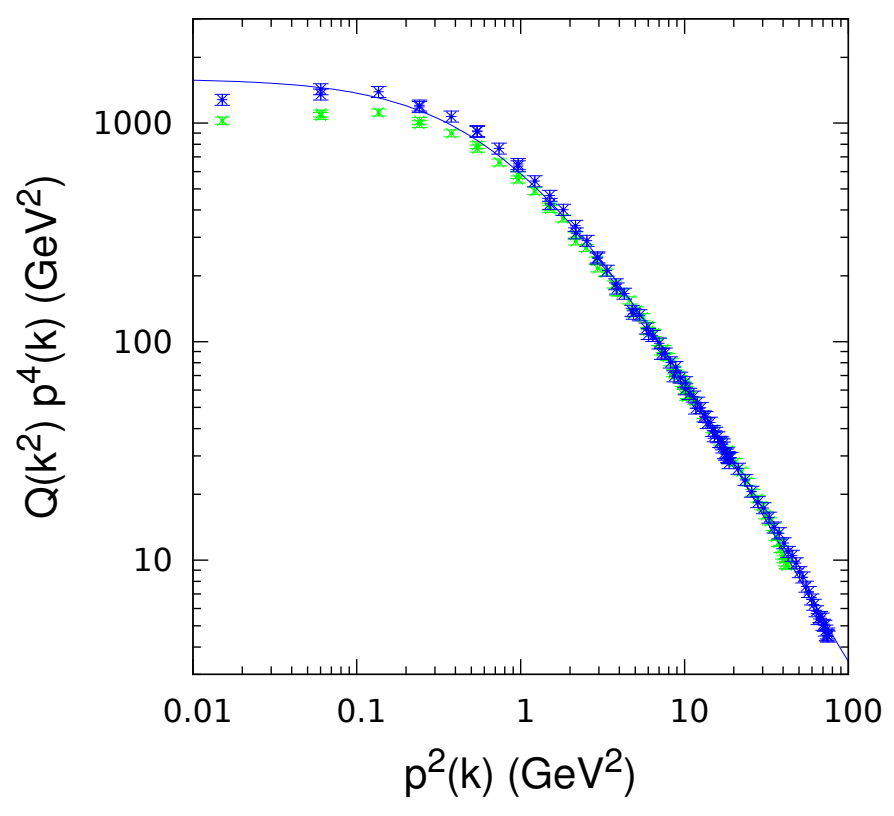

Figure 2: Data for $\beta=2.34940204$ and $V=72^{4}(\times)$ matched $[17,18]$ with data $\beta=2.43668228$ and $V=96^{4}(*)$, fitted using Eq. (3.2) with $t=3.0(0.2)\left(\mathrm{GeV}^{2}\right), u=3.9(0.3)(\mathrm{GeV}), s=58.0(9.8)\left(\mathrm{GeV}^{2}\right)$ and $c=247(16)$.

\section{References}

[1] A. Cucchieri, D. Dudal, T. Mendes and N. Vandersickel, Phys. Rev. D90, 051501 (2014).

[2] G. B. West, Phys. Lett. B115, 468 (1982).

[3] K. G. Wilson, Phys. Rev. D10, 2445 (1974).

[4] S. Mandelstam, Phys. Rev. D20, 3223 (1979).

[5] M. Baker, J. S. Ball and F. Zachariasen, Nucl. Phys. B186, 531 (1981).

[6] M. Baker, J. S. Ball and F. Zachariasen, Nucl. Phys. B186, 560 (1981).

[7] J. M. Cornwall, Phys. Rev. D26, 1453 (1982).

[8] M. Stingl, Phys. Rev. D34, 3863 (1986) [Erratum-ibid. D36, 651 (1987)].

[9] V. N. Gribov, Nucl. Phys. B 139, 1 (1978).

[10] L. Giusti, M. L. Paciello, C. Parrinello, S. Petrarca and B. Taglienti, Int. J. Mod. Phys. A16, 3487 (2001).

[11] N. Vandersickel and D. Zwanziger, Phys. Rept. 520, 175 (2012).

[12] S. P. Sorella, Phys. Rev. D80, 025013 (2009).

[13] J. A. Gracey, Eur. Phys. J. C70, 451 (2010).

[14] D. Zwanziger, arXiv:0904.2380 [hep-th].

[15] S. Furui, PoS LAT 2009, 227 (2009). 
[16] D. Zwanziger, Phys. Rev. D81, 125027 (2010).

[17] D. B. Leinweber et al., Phys. Rev. D60, 094507 (1999) [Erratum-ibid. D61, 079901 (2000)].

[18] A. Cucchieri, T. Mendes and A. R. Taurines, Phys. Rev. D67, 091502 (2003).

[19] J. A. Gracey, JHEP 1002, 009 (2010). 